\title{
Resonant tunneling through a small quantum dot coupled to superconducting leads
}

\author{
A. Levy Yeyati, J.C. Cuevas, A. López-Dávalost and A. Martín-Rodero \\ Departamento de Física Teórica de la Materia Condensada $C$-V. \\ Universidad Autónoma de Madrid, E-28049 Madrid, Spain.
}

\begin{abstract}
We address the problem of non-linear transport through discrete electronic levels in a small quantum dot coupled to superconducting electrodes. In our approach the low temperature $I-V$ characteristics can be calculated including all multiple quasi-particle and Andreev processes. The limit of very weak coupling to the leads and large charging energies is briefly analyzed comparing the calculated lineshapes of the $I-V$ curves with recent experimental results. When the coupling to the leads increases and Coulomb blockade effects can be neglected, the combination of multiple Andreev processes and resonant transmission gives rise to a rich subgap structure which largely differs from the one found in the more studied S-N-S systems. We concentrate on this regime showing how multiple processes can be included within a simple sequential tunneling picture qualitatively explaining the subgap structure. We suggest an experimental set-up where the predicted effects could be observed.
\end{abstract}

PACS numbers: 74.50.+r, 73.40.Gk, 73.20.Dx

Resonant tunneling through small systems characterized by discrete electronic states weakly coupled to metallic leads has been the object of extensive studies for nearly a decade [1]. This physical situation is, in a sense, common to a large variety of systems ranging from semiconductor quantum dots [2], small metallic islands [3] or atomic impurities [团, which can exhibit characteristic phenomena like Coulomb-blockade and the Kondo effect. More 
recently there has been a growing interest in the special case in which these systems include superconducting parts bringing the possibility of observing new effects associated with the superconducting state. While a large part of these efforts have been devoted to understand the interplay between charging and paring effects in "large" mesoscopic superconducting islands with a mean level spacing much smaller than the energy gap [5], less attention has been paid to the case where discrete levels can be resolved [6]. In this communication we shall consider this latter situation for the case where both leads are superconducting.

A system of this kind has been recently investigated experimentally by Ralph et al. who could resolve individual electronic states in the tunneling through a nanometer $\mathrm{Al}$ particle weakly coupled to superconducting leads [7]. For these small particle sizes and extremely weak coupling, both the charging energy, $E_{C}$, and the single-particle level spacing, $\delta$, are much larger than the gap parameter of the superconducting leads, $\Delta$. In this regime the contribution of Andreev processes can be shown to be negligible and only single quasiparticle processes need to be considered. On the other hand, for a system with a larger coupling to the leads and smaller charging energies, multiple Andreev reflections (MAR) may become very important for determining the transport properties. One would expect that the combination of MAR with resonant transmission through the discrete levels would give rise to a rich subgap structure (SGS) in the $I-V$ characteristics, containing new features when compared to the more studied case of S-N-S junctions. An ideal experimental set-up for exploring these effects would be one in which both the resonant level position and the coupling to the leads could be modified in a controlled way like in a normal artificial atom [2]. This situation is represented schematically in Fig. 1.

The aim of this communication is to give a theoretical analysis of the non-linear dc characteristics for systems of the kind discussed above. The analysis is non-perturbative in the coupling to the leads and therefore valid both for the weak and strong coupling regimes. In the weak coupling case our theory naturally explains the observed lineshapes [7] without the need to resort to a phenomenological broadening of the BCS density of states. We shall mainly concentrate in the case where the resonant level lies within the energy gap region 
and the Coulomb blockade can be neglected, for which a novel subgap structure is predicted to appear.

As mentioned above, a small quantum dot connected to superconducting leads is characterized by the interplay of many different energy scales. In order to analyze in detail the basic phenomena that can be observed in the case of large mean level spacing $(\delta \gg \Delta)$, we shall restrict the present theoretical discussion to the simplest case where transport takes place through a single resonant level. For describing this physical situation we use the following model Hamiltonian

$$
\hat{H}=\hat{H}_{L}+\hat{H}_{R}+\sum_{\nu, \sigma} t_{\nu}\left(\hat{c}_{\nu \sigma}^{\dagger} \hat{c}_{0 \sigma}+\hat{c}_{0 \sigma}^{\dagger} \hat{c}_{\nu \sigma}\right)+\sum_{\sigma} \epsilon_{0} \hat{n}_{0 \sigma}+U \hat{n}_{0 \uparrow} \hat{n}_{0 \downarrow}
$$

where $\hat{H}_{L}$ and $\hat{H}_{R}$ are BCS Hamiltonians describing the left and right superconducting leads, characterized by gap parameters $\Delta_{L}=\Delta_{R}=\Delta ; \epsilon_{0}$ is the bare resonant level position, $t_{\nu}$ with $\nu=L, R$ are hopping parameters which connect the level to the left and right leads, and the $U$ term describes the intralevel Coulomb repulsion. This parameter is basically the dot charging energy, $E_{C}$, and is related to the total dot capacitance $C$, by $U \sim e^{2} / 2 C$ [ 8 . For the case of superconducting leads it is convenient to choose a gauge in which an applied bias voltage is introduced through time dependent phase factors modulating the hopping parameters [9] as $t_{\nu} \rightarrow t_{\nu} \exp \left(i e V_{\nu} \tau / \hbar\right)$, where $V_{\nu}$ are the voltage drops between the leads and the central region. Hamiltonian (1) is nothing but an Anderson model which has been extensively used for studying the case of a small dot coupled to normal leads [10]. For the subsequent discussion it is convenient to introduce the normal elastic tunneling rates $\Gamma_{\nu}=\pi\left|t_{\nu}\right|^{2} \rho_{\nu}(\mu)$, where $\rho_{\nu}(\mu)$ are the normal spectral densities of the leads at the Fermi level.

There are two different regimes in which correlation effects associated with the $U$-term in Hamiltonian (1) can either be neglected or taken into account in a simple way, and yet lead to a non-trivial behavior. The first regime corresponds to a case where the couplings to the leads are not extremely small and the dot capacitance is large enough as to smear out the Coulomb blockade effect $\left(\Gamma_{L, R} \sim U\right)$. In this case the system behaves as if there 
were a single spin-degenerate resonant level at $\epsilon \simeq \epsilon_{0}+U<n_{0}>$, i.e. a restricted Hartree approximation on the $U$-term would be reasonable. When this effective level lies within the superconducting gap both single quasiparticle and Andreev processes give an important contribution to the subgap $I-V$ structure. The second regime would correspond to the experimental conditions of Ref. [7] in which $U \gg \Delta \gg \Gamma_{L, R}$. Double occupancy of the resonant level becomes then very unlikely, Andreev processes are strongly suppressed and thus only single-quasiparticle processes have to be considered. This situation can be simply simulated by replacing the isolated dot Hamiltonian by a single non-degenerate effective level. The analysis of other regimes, where correlations effects could play a relevant role (like for instance in the Kondo effect) would be given in a forthcoming publication.

The transport properties of this model can be obtained using the same approach as in Refs. [9] which is based on non-equilibrium Green functions techniques. As discussed in these references, this method is based on an expansion of the relevant Green functions in terms of the hopping elements coupling the dot to the leads. When both electrodes are superconducting, due to the presence of MAR, this expansion leads to an infinite set of algebraic equations for the Green functions, which can be solved using recursive techniques as discussed in detail in Refs. [9]. As a consequence of this infinite series of MAR, the average current, $I(\tau)$, contains all harmonics of the Josephson frequency $\omega_{0}=2 \mathrm{eV} / \hbar$, i.e. the current can be written as $I(\tau)=\sum_{n} I_{n} \exp \left(i n \omega_{0} \tau\right)$. We shall concentrate here in its dc part $I_{0}$. Further details of this formalism, as applied to the present case, will be given elsewhere.

Let us first analyze the simplest case $U \gg \Delta \gg \Gamma$. As mentioned above the theory is then greatly simplifyed by suppression of Andreev reflections. The dc current is then given by the resonant tunneling like expression

$$
I_{0}(V)=\frac{4 e}{h} \int_{-\infty}^{\infty} d \omega \frac{\Gamma_{L}^{S}(\omega) \Gamma_{R}^{S}(\omega)}{(\omega-\epsilon)^{2}+\left[\Gamma_{L}^{S}(\omega)+\Gamma_{R}^{S}(\omega)\right]^{2}}\left[n_{F}(\omega-e V / 2)-n_{F}(\omega+e V / 2)\right],
$$

where the superconducting tunneling rates $\Gamma_{L, R}^{S}$ are defined as $\Gamma_{L, R}^{S}(\omega)=\Gamma_{L, R} \tilde{\rho}^{S}(\omega \pm e V / 2)$, $\tilde{\rho}^{S}$ being the corresponding dimensionless BCS spectral density given by $\tilde{\rho}^{S}=|\omega| / \sqrt{\omega^{2}-\Delta^{2}}$, 
and $n_{F}(\omega)$ is the Fermi distribution function. In this expression $\epsilon$ is the effective resonant level position in which the charging effects have been included.

In Fig. $2 I_{0}(V)$ is shown for decreasing coupling to the leads. Notice that the lineshape progressively resembles a BCS spectral density in agreement with the experimental observations [7]. In Ref. [7] special attention was given to the behavior of the $I-V$ characteristics near the threshold voltage, in particular, to the broadening and reduced amplitude with respect to a simple BCS spectral density. In fact, our theory predicts a finite height and width of these resonances without having to resort to the introduction of any phenomenological broadening parameter. This is a simple consequence of having a small but finite coupling to the leads. Eq. (2) in the limit of vanishing coupling reduces to $I_{0}(V) \sim \Gamma_{L}^{S}(\epsilon) \Gamma_{R}^{S}(\epsilon) /\left(\Gamma_{L}^{S}(\epsilon)+\Gamma_{R}^{S}(\epsilon)\right)$, which coincides with the result one would expect from a sequential tunneling picture [11]. The coherent processes taken into account in Eq. (2) are responsible for the rounding off of the resonant peaks, as illustrated in Fig. 2. We have estimated that the experimental situation in Ref. [7] would approximately correspond to the case plotted as a full line in Fig. 2 .

Let us next consider the case where $U \sim \Gamma_{L, R}$, which, as discussed above, can be described by a single spin-degenerate effective level. In contrast to the previous case, now the effect of MAR become crucial and the full formalism is needed for the calculation of the current. The importance of MAR in this case is clearly displayed in the subgap structure exhibited in the $I-V$ curves shown in Fig. 3. This figure corresponds to a symmetric case where the level position $\epsilon$, is fixed at $\epsilon=0$, while $\Gamma=\Gamma_{L}=\Gamma_{R}$ varies between $\Gamma \gg \Delta$ and $\Gamma<\Delta$. As can be observed, in the limit $\Gamma \gg \Delta$ SGS is absent, the relevant feature being a saturation of the current at $I_{0} \sim 4 e \Delta / h$ for $V \rightarrow 0$. This feature is commonly referred to as a "foot" in the context of S-N-S structures [12]. In this regime we recover the result for a ballistic single-mode superconducting quantum point contact as obtained recently by different authors [9]:13]. The departure from the S-N-S behavior becomes more apparent for decreasing $\Gamma$, where a progressively pronounced SGS appears. Notice that, in contrast to what is found in S-N-S structures with a continuum of states in the central region, in the 
present case the $I_{0}(V)$ curve itself exhibits oscillations with the concomitant appearance of negative differential conductance.

The particular SGS found in this system is related to the influence of resonant tunneling on the MAR processes. In fact, in the limit of $\Gamma \ll \Delta$ the position and shape of the subgap current peaks can be understood by means of the following simple picture. When $e V<2 \Delta$ current between the superconducting leads can flow due to MAR processes. As in the case of S-S or S-N-S junctions, there appear jumps in the subgap $I-V$ characteristics at voltages corresponding to the opening of a new Andreev channel. However, the amplitude of these subgap processes is greatly modified by the presence of a resonant level between the leads, in such a way that only those MAR "trajectories" that connect the resonant level to the leads spectral densities give a significant contribution to the current. The inset in Fig. 4a illustrates the 2nd order Andreev trajectory which gives the dominant contribution to the current when $2 \Delta / 3<e V<2 \Delta$. As $e V$ decreases towards $2 \Delta / 3$, the energies of the initial and final states on this trajectory approach the gap edges, which results in a BCS DOS-like shape of the current peak at $e V=2 \Delta / 3$ (see Fig. 4a).

This simple picture enables us to evaluate the SGS at $\Gamma \ll \Delta$ analytically. The first step is to identify the generalized tunneling rates $\Gamma_{n}^{(i n)}(\omega)$ and $\Gamma_{n}^{(o u t)}(\omega)$ associated with the $n$ order Andreev process connecting the dot to the leads. Here the $\Gamma_{n}^{(i n)}$ 's give the probability of an electron or a hole to get into the dot as an electron, while the $\Gamma_{n}^{(\text {out })}$ 's correspond to the complementary processes where one electron leaves the dot and reaches the leads as an electron or as a hole. A simple analysis yields, for the symmetric case $\Gamma=\Gamma_{L}=\Gamma_{R}$, the following expressions

$$
\Gamma_{n}^{(o u t, i n)}(\omega)=\Gamma^{2 n+1} \tilde{\rho}^{S}(\omega \pm(2 n+1) e V / 2) \prod_{j=1}^{n}\left|\frac{f(\omega \pm(2 j-1) e V / 2)}{\omega-(-1)^{j} \epsilon \pm j e V}\right|^{2}
$$

where $f(\omega)$ is the dimensionless BCS pairing amplitude of the uncoupled leads defined as $f(\omega)=\Delta / \pi \sqrt{\Delta^{2}-\omega^{2}}$. Notice that $\Gamma^{2}|f(\omega)|^{2}$ is the Andreev reflection probability at the lowest order in $\Gamma$, while the denominator in Eq. (3) is related to the transmission probability through the dot. The total current can then be computed as the sum of the contributions due 
to all possible combinations of in and out processes. Every contribution must be weighted by the total charge which is transferred in the combined process. For instance, the process depicted as an inset in Fig. 4a is a combination of two processes of the first order and has an associated charge of $3 e$. The resulting expression for the current is

$$
I_{0}(V)=\frac{8 e \pi}{h} \sum_{n, m}(n+m+1) \frac{\Gamma_{n}^{(i n)}(\epsilon) \Gamma_{m}^{(o u t)}(\epsilon)}{\Gamma_{n}^{(i n)}(\epsilon)+\Gamma_{m}^{(o u t)}(\epsilon)} .
$$

Fig. 4 illustrates in further detail the SGS of the $I-V$ curves for $\Gamma \ll \Delta$. For comparison the results given by Eq. (4) are also shown. As can be observed, this simple approximation fairly reproduces the exact numerical results in this small $\Gamma$ limit. The rounding off of the peaks which is observed for increasing $\Gamma$ (see Fig. 3) is due to off resonant processes as discussed for the case of large charging energy. In the limit $\Gamma \sim \Delta$ the sequential tunneling picture breaks down due to the interference among the different multiple processes.

For the general case with $\epsilon \neq 0$, the SGS becomes more complex due to the appearance of additional resonances. This is illustrated in Fig. 4b for the case $\epsilon=0.2 \Delta$ and $\Gamma=5 \times 10^{-2} \Delta$. As can be deduced from the sequential formulae of Eq. (4), resonances appear both at $e V_{n}^{+}=2(\Delta+\epsilon) /(2 n+1)$ and $e V_{n}^{-}=2(\Delta-\epsilon) /(2 n+1)$ corresponding to processes in which the inital or final states are at the gap edges. The sequential picture also predicts the appearance of resonances at $j e V=2 \epsilon$ due to resonant coupling between electron and hole states. In the exact numerical results the resonances are somewhat shifted with respect to these predictions and some of them are difficult to resolve. Nevertheless, it should be stressed that the main qualitative features of the exact SGS are already contained in Eq. $(4)$.

As has been already mentioned, an ideal experimental set-up to study the interplay between resonant tunneling and MAR would be an "artificial-atom" with superconducting leads as represented in Fig. 1. In this type of structure, for a quantum dot area of $\sim$ $(100 \mathrm{~nm})^{2}$, the mean level spacing would be around $2.7 \mathrm{meV}$ [8], which is much larger than the superconducting gap on the leads if these were made of $A l\left(\Delta_{A l} \sim 0.18 \mathrm{meV}\right)$. On the other hand, the coupling to the leads could be given any desired value by varying the 
conductance of the two point contacts, and the charging energy could also in principle be changed by varying the different capacitances between the dot and the surrounding metallic leads and gates. Thus, the conditions for observing the $\Gamma \sim U<\Delta$ could be attainable. In fact, a system of these characteristics would not be very different from the superconducting quantum point contact developed by Takayanagi et al. [14].

In conclusion, we have presented model calculations for the transport through discrete resonant levels coupled to superconducting leads. We have briefly analyzed the case corresponding to a large charging energy and a extremely weak coupling, finding good agreement with the experimental results of Ref. [0]. The main part of this work has been concentrated in the study of the case $U \sim \Gamma<\Delta$, where Coulomb blockade effects can be neglected. For this regime we have shown how the interplay between resonant tunneling and multiple Andreev reflection processes give rise to a novel subgap structure in the $I-V$ curves, which could be in principle detected if the adequate conditions are met in the experiments. We have shown how the effects of MAR can be taken into account in a sequential tunneling picture by the introduction of generalized tunneling rates. This opens the possibility of analyzing more complex situations in which the mean level spacing is comparable to the superconducting gap and multiple resonances are involved. Work along these lines is under progress.

Support by Spanish CICYT (Contract No. PB93-0260). One of us (A.L.D.) acknowledges partial financial support by "Instituto Nicolás Cabrera". 


\section{REFERENCES}

* Present address: Instituto Balserio-Centro Atómico Bariloche, Comisión Nacional de Energía Atomica, Bariloche, Argentina.

[1] M.A. Kastner, Rev. Mod. Phys. 64, 849 (1992).

[2] L.P. Kouwenhoven et al., Z. Phys. B 85, 367 (1991); U. Meirav et al., Phys. Rev. Lett. 65, $771(1990)$.

[3] T.A. Fulton et al., Phys. Rev. Lett. 59, 109 (1987).

[4] D.C. Ralph et al., Phys. Rev. Lett. 72, 3401 (1994).

[5] P. Lafarge et al., Phys. Rev. Lett. 70, 994 (1993); M.T. Tuominen et al., Phys. Rev. B 47, 11599; K.A. Matveev et al., Phys. Rev. Lett. 70, 2940 (1993).

[6] L.I. Glazman et al., JETP Lett. 49, 659 (1989); V.A. Khlus et al., Physica C 214, 413 (1993); C.W.J. Beenakker and H. van Houten, in Single Electron Tunneling and Mesoscopic Devices (Springer, Berlin, 1992).

[7] D.C. Ralph et al., Phys. Rev. Lett. 74, 3241 (1995).

[8] H. van Houten et al., in Single Charge Tunneling, edited by H. Grabert and M.H. Devoret (Plenum, New York, 1992).

[9] J.C. Cuevas et al. Phys. Rev. B 54, 7366 (1996). A. Levy Yeyati et al., Phys.: Condens. Matter 8, 449 (1996); A. Martín-Rodero et al., Physica B 218, 126 (1996).

[10] S. Hershfield et al., Phys. Rev. Lett. 67, 3720 (1991); Y. Meir et al., Phys. Rev. Lett 70, 2601 (1993); A. Levy Yeyati et al., Phys. Rev. Lett. 71, 2991 (1993); C.A. Stafford and Das Sarma, Phys. Rev. Lett. 72, 3590 (1994).

[11] D.V. Averin et al., Phys. Rev. B 44, 6199 (1991); C.B. Whan and T.P. Orlando condmat 9607095). 
[12] U. Gunsenheimer et al., Phys. Rev. B 50, 6317 (1994).

[13] D. Averin, and A. Bardas, Phys. Rev. Lett. 75, 1831 (1995); M. Hurd, S. Data, and P.F. Bagwell, unpublished.

[14] H. Takayanagi et al., Phys. Rev. Lett. 75, 3533 (1995). 


\section{FIGURES}

FIG. 1. Schematical representation of a quantum dot defined in a 2D electron gas and coupled to superconducting leads

FIG. 2. Zero temperature $I-V$ characteristics corresponding to the case of large charging energy and extremely small coupling. Full line for $\Gamma_{L}=5 \times 10^{-3} \Delta$, dashed line for $\Gamma_{L}=10^{-3} \Delta$ and dotted line for $\Gamma_{L}=2 \times 10^{-4} \Delta$. In all cases $\Gamma_{R}=4 \Gamma_{L}$ and $\epsilon=5 \Delta$.

FIG. 3. Zero temperature $I-V$ characteristics corresponding to the case where Coulomb blockade is absent for different values of $\Gamma=\Gamma_{L}=\Gamma_{R}$. The effective resonant level is $\epsilon=0$.

FIG. 4. Detail of the subgap structure for $\Gamma=5 \times 10^{-2} \Delta$ with $\epsilon=0$ (a) and $\epsilon=0.2 \Delta$ (b). Full line: complete numerical calculation; dotted line: sequential approximation discussed in the text. The inset represents a typical resonant "trajectory" mediated by two Andreev reflections. The arrows indicate the position of the first resonances for $\epsilon=0.2 \Delta$. 


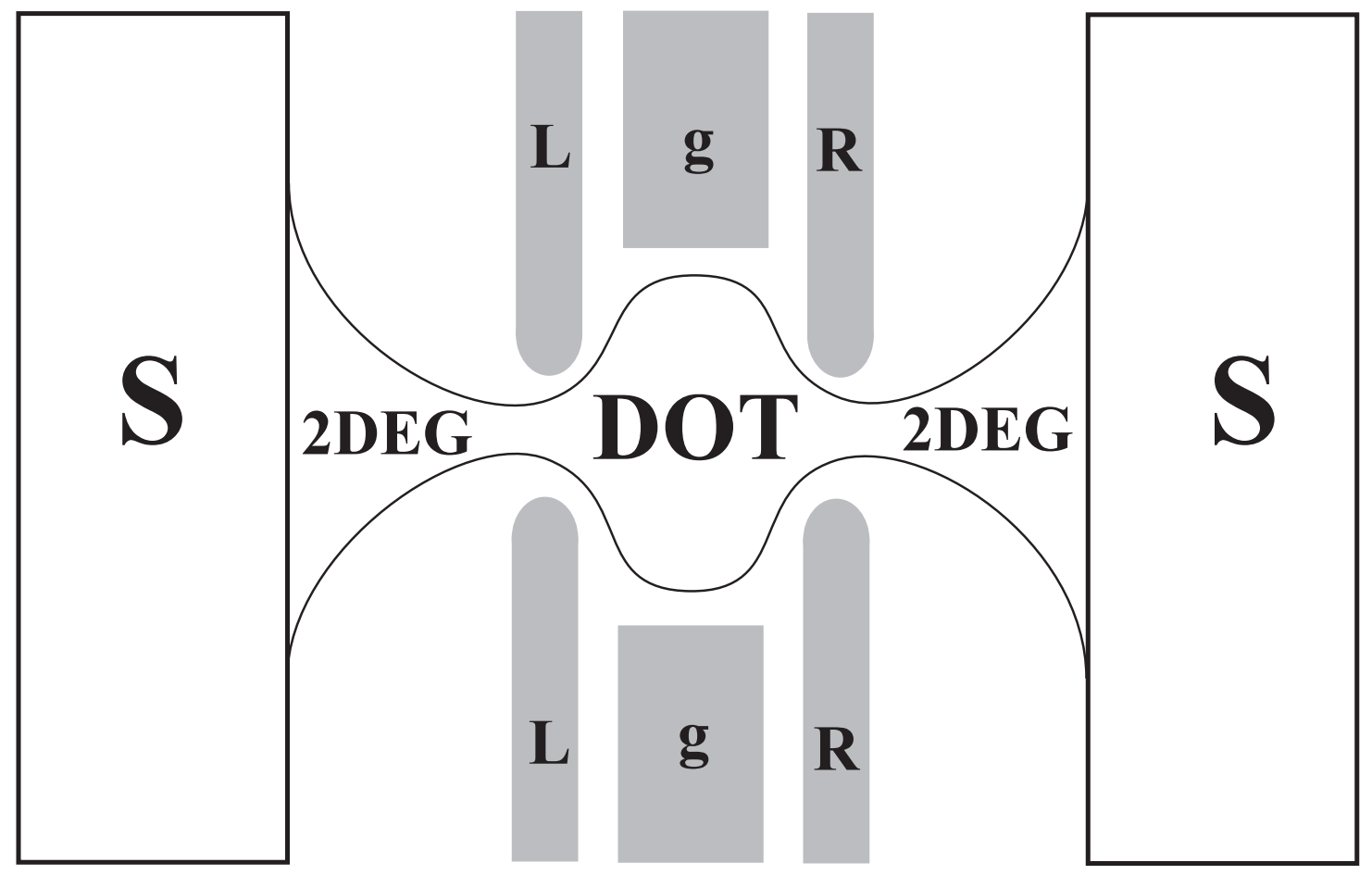




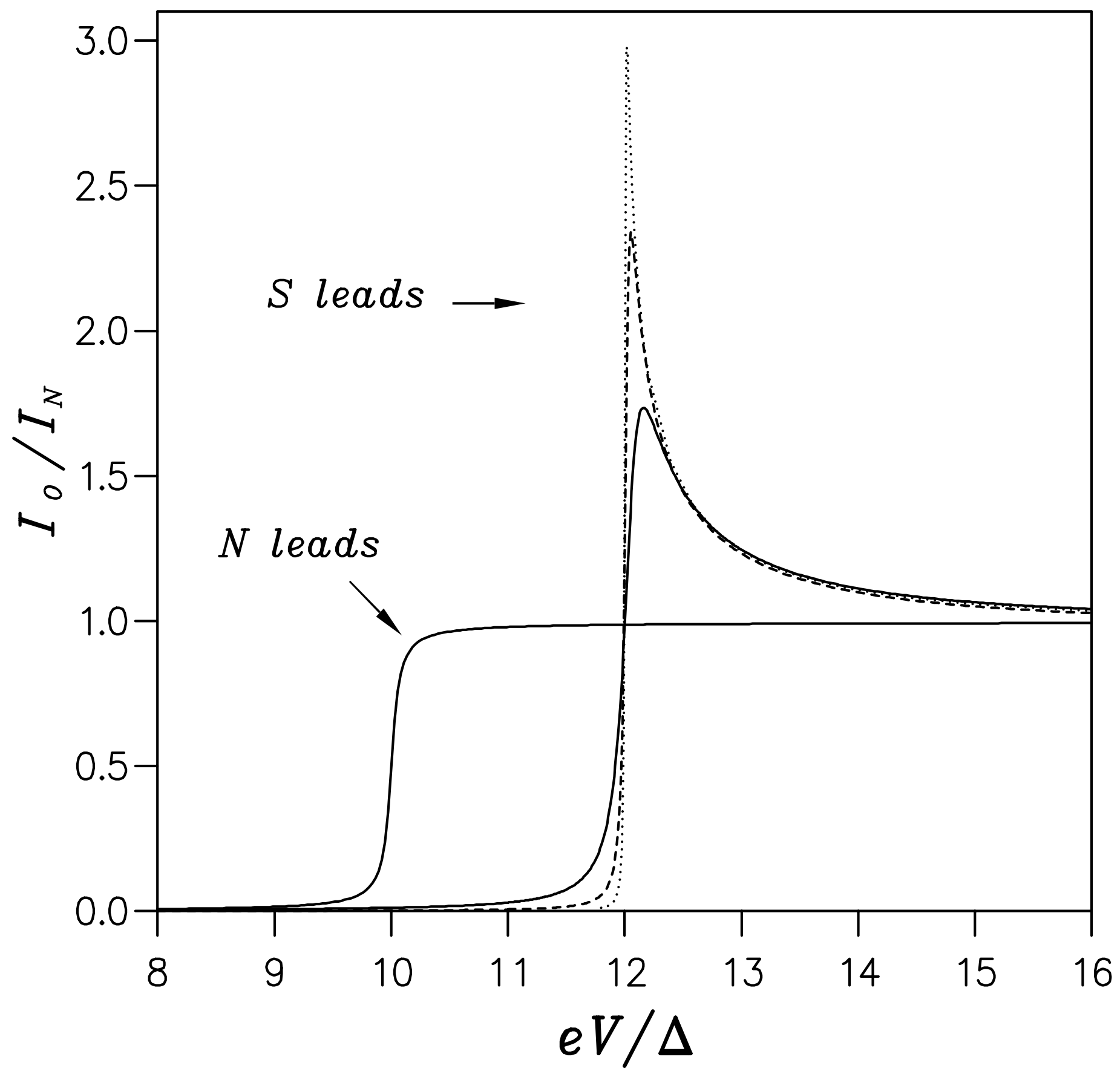




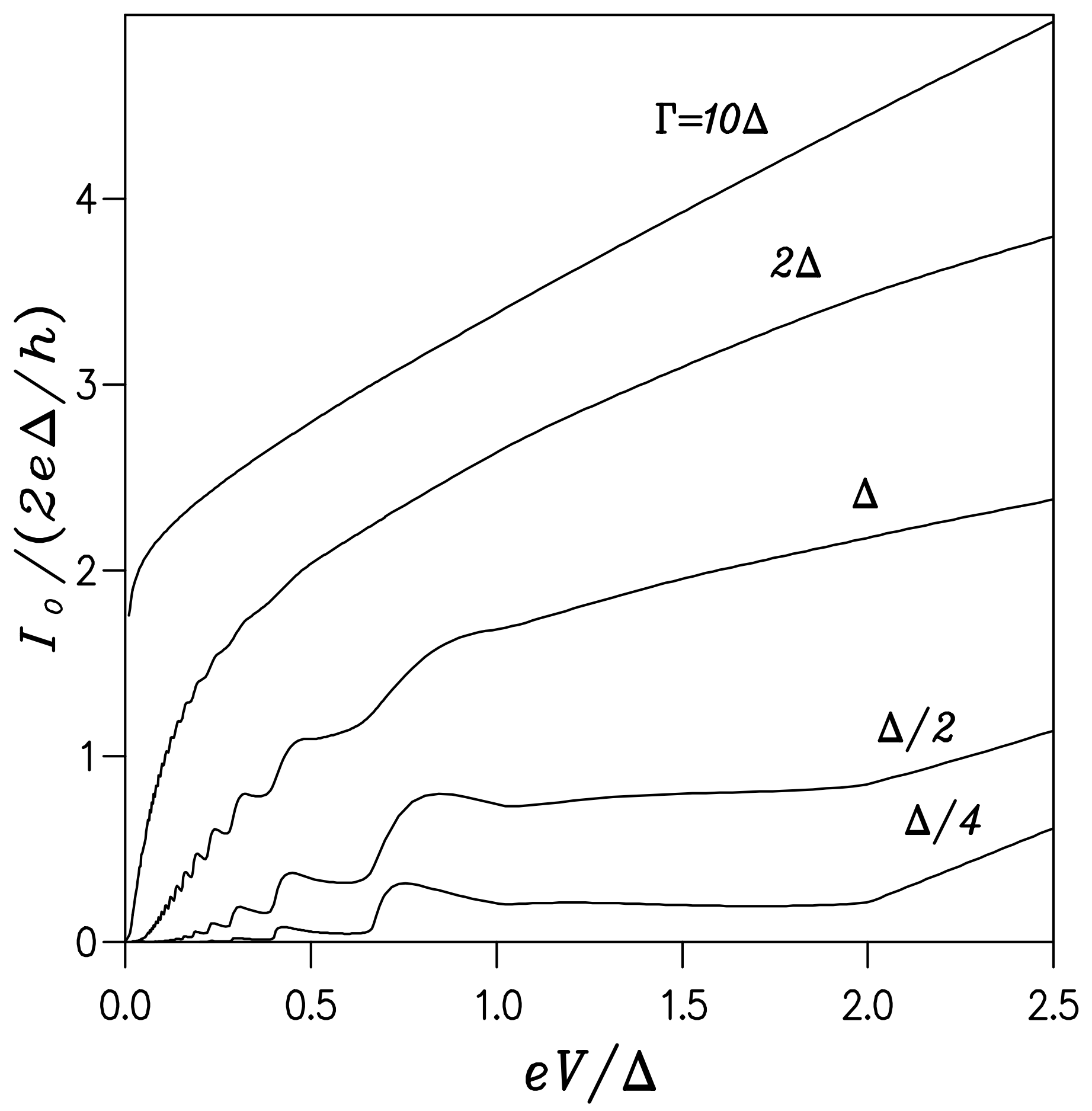




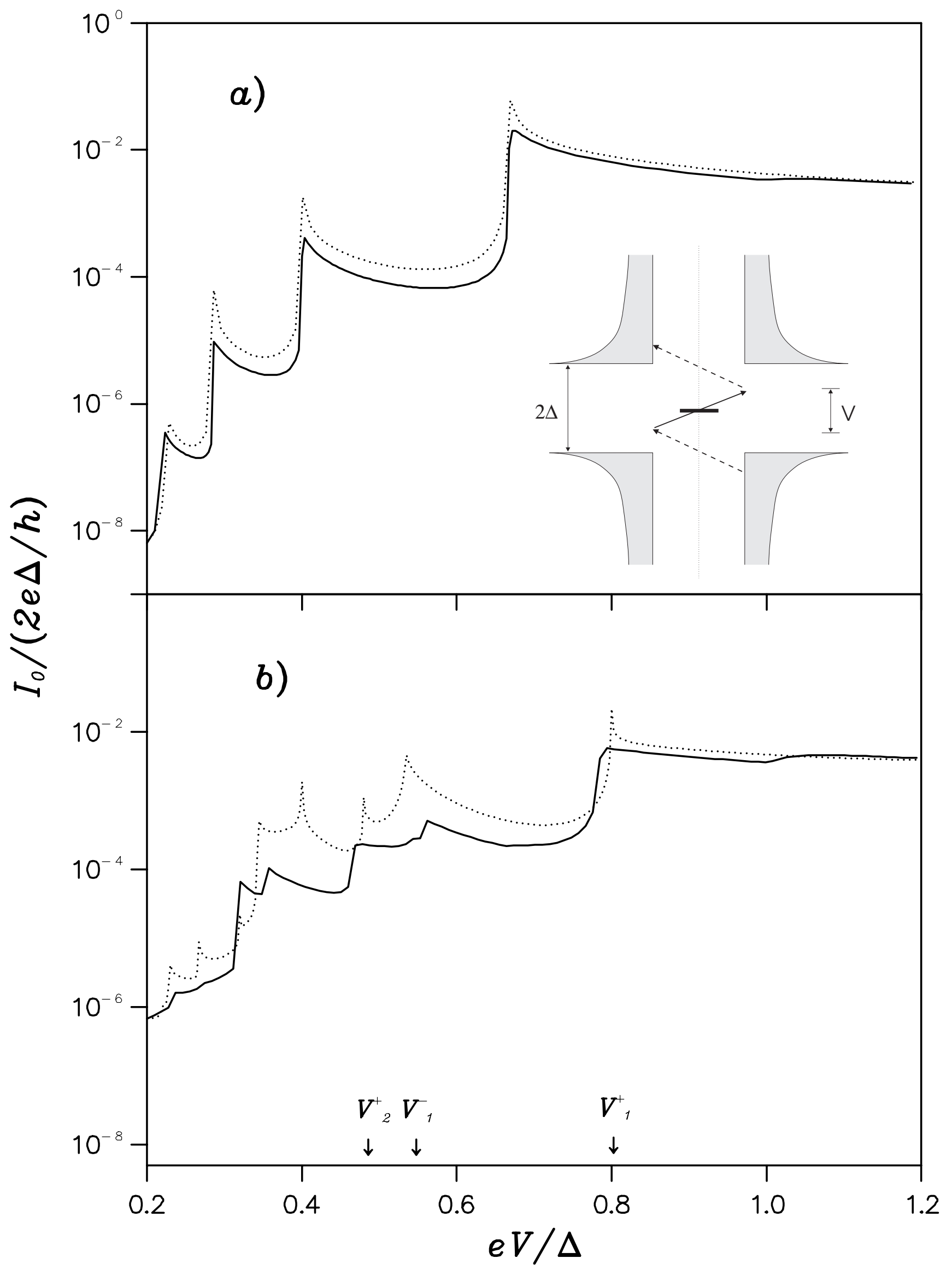

